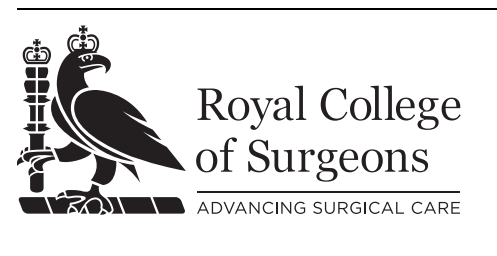

\title{
GENERAL SURGERY
}

\section{Toxicity and survival of anal cancer patients treated with intensity-modulated radiation therapy}

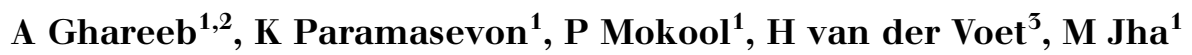 \\ ${ }^{1}$ Department of General Surgery, James Cook University Hospital, Middlesbrough, UK \\ ${ }^{2}$ Institute of Genetic Medicine, Newcastle University, International Centre for Life, \\ Newcastle upon Tyne, UK \\ ${ }^{3}$ Cancer Services, James Cook University Hospital, Middlesbrough, UK
}

ABSTRACT

INTRODUCTION The definitive treatment of anal cancer with chemoradiotherapy spares abdominoperineal resection for salvage treatment but carries a high burden of toxicity. Intensity-modulated radiation therapy has been implemented to reduce toxicity, reduce treatment breaks and improve survival. However, large and long-term studies are lacking. We aimed to investigate the toxicities and long-term survival of anal cancer patients treated with intensity-modulated radiation therapy at James Cook University Hospital, Middlesbrough.

MATERIALS AND METHODS We conducted a retrospective analysis of all patients with squamous cell anal cancer treated at James Cook University Hospital between July 2010 and April 2017. All patients were uniformly treated with intensitymodulated radiation therapy-based chemoradiation with curative intent. A subset of these patients was followed-up prospectively by an oncologist for acute and late toxicity. We calculated Kaplan-Meier estimates of survival statistics and compared our results with those of previous trials which used conventional radiotherapy.

RESULTS We studied 132 patients, including a toxicity subset of 64, for a median follow-up time of 43 months (range 3-84 months). Eleven patients (8.3\%) underwent salvage abdominoperineal resection. Grade 3+ acute non-haematological, gastrointestinal, genitourinary and dermatological toxicity were found in $56.2 \%, 12.3 \%, 0 \%$ and $50.7 \%$ of the toxicity subset $(n=64)$. Median treatment duration was 37 days. Overall and colostomy-free survival at five years were $68.3 \%$ and $85.3 \%$, respectively. Tumour size $(P=0.006)$ and age $(P=0.002)$ predicted shorter overall survival.

CONCLUSIONS Intensity-modulated radiation therapy probably reduces acute gastrointestinal and genitourinary toxicity compared with conventional radiotherapy, while resulting in similar overall and colostomy-free survival. We suggest that further dose escalation may improve survival in patients with T3/T4 tumours.

\section{KEYWORDS}

Anal cancer - Intensity-modulated radiation therapy - Chemoradiation - Abdominoperineal resection - Survival - Toxicity

Accepted 7 October 2018

CORRESPONDENCE TO

Ali Ghareeb, E: Ali.ghareeb@newcastle.ac.uk

\section{Introduction}

Anal cancer is a rare squamous-cell carcinoma, with approximately 27,000 cases recorded worldwide in $2008 .^{1}$ A 2017 epidemiological study shows that the incidence is on the rise in both sexes, with associated risk factors including human papillomavirus (HPV), HIV and risky sexual behaviours. ${ }^{2}$ The majority of anal cancers are caused by HPV-16. ${ }^{1,3,4}$ It is also estimated to be nine times higher in HIV-positive men who have sex with men than HIV-negative who have sex with men. ${ }^{5}$

Since the work of Norman Nigro at Wayne State University Hospital, ${ }^{6-8}$ chemoradiotherapy has been established as the primary treatment, with abdominoperineal resection reserved as salvage treatment. ${ }^{9}$
Several randomised controlled trials have since confirmed the supremacy of the original regimen over others. ${ }^{10-14}$ Chemoradiotherapy offers a good prospect of cure with reduced morbidity and improved sphincter preservation. In most patients, chemoradiotherapy precludes the need for permanent colostomy. However, this treatment is not free from serious adverse effects, which include acute dermatological, gastrointestinal, haematological and cardiovascular toxicities. Large studies have demonstrated high levels of toxicity related to chemoradiotherapy. ${ }^{11,12,15}$ In the ACT II study, which examined cisplatin compared with mitomycin chemoradiotherapy, $62 \%$ of patients in the mitomycin group experienced an acute grade 3 or 4 nonhaematological toxicity. ${ }^{11}$ 
Intensity-modulated radiation therapy (IMRT) has been introduced to mitigate some of these untoward effects by reducing the radiation exposure of organs at risk. Its efficacy in the treatment of anal cancer has only been demonstrated in a number of small prospective and retrospective studies, ${ }^{16-22}$ and long-term survival data are limited.

Our study was conducted in a tertiary referral centre the sole provider of treatment for anal cancer to a population of 1.5 million in the North-East of England. ${ }^{23}$ Since July 2010, all anal cancer patients at James Cook University Hospital, Middlesbrough, have been treated exclusively with IMRT. We aimed to study the toxicity experienced by patients treated for anal cancer at our hospital, their longterm survival and the frequency of salvage abdominoperineal resection to determine the relative benefits of IMRT compared with conventional radiotherapy.

\section{Materials and methods \\ Study design}

We retrospectively collected data on the demographics, TNM staging, survival and disease recurrence of patients with anal cancer. Data had been recorded in hospital databases by clinicians during episodes of routine care. From July 2010, a prospective patient subset was created under a designated consultant oncologist to monitor treatmentrelated toxicity closely. The patients in this toxicity subset were selected only because they had been treated by the oncologist collecting toxicity data and not because of any disease or patient-related factors. Patients in the prospective toxicity subset were followed up routinely. This involved three-monthly follow-up for the first two years following treatment and six-monthly follow-up thereafter until five years after treatment.

\section{Study participants}

All patients diagnosed with an anal malignancy between July 2010 and April 2017 who were subsequently referred to our hospital for further investigations and treatment were added to the hospital's cancer registry. Following histological clarification, only patients with a diagnosis of squamous cell carcinoma were retained for further analysis.

\section{Treatment}

Prior to July 2010, patients were treated with conventional radiotherapy as per the ACT II protocol. ${ }^{11}$ After the installation of a TomoTherapy ${ }^{\circledR}$ machine in the oncology department in 2010, an IMRT protocol was developed. Volumetric modulated arc therapy became available in October 2012 and both forms of IMRT were used subsequently. All patients included in this study received IMRT-based chemoradiation with curative intent. For T1/2 tumours a standard regimen of 50.4 Gy and 42 Gy in 28 fractions were prescribed for the main tumour and nodal volume, respectively. For T3/4 tumours, 54 Gy to the main tumour volume and $45 \mathrm{~Gy}$ to the nodal volume in 30 fractions for uninvolved nodes were prescribed, and 50.4 Gy in 30 fractions to involved lymph nodes if $3 \mathrm{~cm}$ or less, or $54 \mathrm{~Gy}$ in 30 fractions if greater than $3 \mathrm{~cm}$.

\section{Data collection}

Patient data were collected from WebICE, the hospital's electronic database. For the patients who formed the prospectively collected toxicity subset, the severity of toxicities was assigned a grade according to the Common Terminology Criteria for Adverse Events (version 4.0). ${ }^{24}$ Vital status, including mortality of participants, was recorded from WebICE. Overall survival and colostomy-free survival were defined from the date of diagnosis, while disease-free survival and metastasis-free survival were defined from the initiation of chemoradiotherapy. Only post-treatment colostomies were counted as events in the colostomy-free survival statistic.

\section{Statistical analysis}

The statistical programming language and environment Rstudio was used for all analyses. We calculated the KaplanMeier estimate for survival curves. Differences between groups were assessed by building a Cox regression model and using the log-rank method to test for significance. The time to metastases, non-parametric survival curve were calculated using interval-censored data. Prognostic data were derived by extrapolating both univariate and multivariate Cox proportional hazards regression models. The proportional hazards assumption held true for each variable tested. Multivariate analysis included tumour size, nodal status, tissue differentiation grading, age at diagnosis and sex.

\section{Results}

A total of 147 patients presented to this unit with an anal malignancy during the period July 2010 to April 2017. Of these patients, 132 had histologically-proven squamous cell carcinoma. Ten patients were diagnosed with adenocarcinoma, two with malignant melanoma, two with neuroendocrine carcinoma and one with adenosquamous carcinoma. Only patients with squamous cell carcinoma were further analysed.

Of those diagnosed with squamous cell carcinoma, $70.5 \%$ were female $(n=93)$ and $29.5 \%$ were male $(n=39)$. The median age at diagnosis was 67 years (range 33-98 years). The results of histopathological and radiological investigations are summarised in Table 1.

\section{Disease progression}

After a median follow-up of 43 months (range 3-84 months), the incidence of locoregional failure was $9.8 \%$ $(n=13)$. Of those patients who developed locoregional failure, 11 had salvage abdominoperineal resection (8.3\%), with a clear resection margin achieved in 8 patients. Two of these patients subsequently died (out-of-hospital deaths of unknown aetiology). Neither patient had been recently admitted to hospital and neither was known to have metastases. Distant metastases were found in 19 patients (14\%), 3 of whom presented at diagnosis. Five patients were given de-functioning stomas for symptom relief. Their primary reasons for de-functioning stoma were impending obstruction $(n=2)$, tenesmus $(n=2)$ and incontinence $(n=1)$. 


\begin{tabular}{|c|c|c|}
\hline & \multicolumn{2}{|c|}{ Patients } \\
\hline & $(n)$ & (\%) \\
\hline \multicolumn{3}{|l|}{ Grade: } \\
\hline 1 & 27 & 20.5 \\
\hline 2 & 52 & 39.4 \\
\hline 3 & 43 & 32.6 \\
\hline Unidentified & 10 & 7.6 \\
\hline \multicolumn{3}{|l|}{ Tumour size: } \\
\hline Tis & 2 & 1.5 \\
\hline $\mathrm{T} 1$ & 24 & 18.2 \\
\hline T2 & 41 & 31.1 \\
\hline T3 & 30 & 22.7 \\
\hline T4 & 35 & 26.5 \\
\hline \multicolumn{3}{|c|}{ Nodal disease stage: } \\
\hline NO & 71 & 53.8 \\
\hline N1 & 26 & 19.7 \\
\hline N2 & 25 & 18.9 \\
\hline N3 & 5 & 3.8 \\
\hline $\mathrm{Nx}$ & 5 & 3.8 \\
\hline \multicolumn{3}{|l|}{ Mets staging: } \\
\hline MO & 129 & 97.7 \\
\hline M1 & 3 & 2.3 \\
\hline
\end{tabular}

All five had stomas performed prior to the initiation of chemoradiotherapy. One further patient was given a de-functioning stoma for symptom relief following the failure of chemoradiotherapy, as she was not fit for salvage abdominoperineal resection.

\section{Survival}

Table 2 summarises the overall survival, colostomy and disease-free survival for this study and compares them to the results of major phase three trials using conventional three-dimensional radiotherapy. Figure 1 shows the Kaplan-Meier survival curves for overall survival, colostomy-free survival and disease-free survival, respectively. Figure 2 shows the time-to-metastasis survival curve for the 19 patients who developed distant metastases. Patients were more likely to present with metastases in the first 12 months after diagnosis than afterwards $(P=0.01)$.

\section{Toxicity}

The three-year overall and colostomy-free survival of the toxicity subset were similar to those of the overall patient cohort (overall survival 78\%; 95\% confidence interval, CI, $68-89 \%$ ), colostomy-free survival $81 \%$ (95\% CI $72-92 \%$, $n=64)$. The median number of days between treatment start and end was 37, while the median number of days on which radiotherapy was received was 30 . Acute toxicity (gastrointestinal, genitourinary or dermatological) of stage 3 or greater was experienced by 34 patients $(53 \%)$, of which nine $(14 \%)$ were grade 4 reactions. Three patients did not receive the prescribed dose of radiation: two had severe acute toxicities, while one did not attend. Twentythree patients $(36 \%)$ had a treatment break of one day or more. Median duration of treatment was 37 days. Overall,

\begin{tabular}{|c|c|c|c|c|c|c|c|c|c|c|c|}
\hline \multirow[t]{2}{*}{ Trial } & \multirow[t]{2}{*}{ Radiotherapy regimen } & \multicolumn{2}{|c|}{$\begin{array}{l}\text { Overall survival } \\
\qquad(\%)\end{array}$} & \multicolumn{2}{|c|}{$\begin{array}{l}\text { Colostomy-free } \\
\text { survival (\%) }\end{array}$} & \multicolumn{2}{|c|}{$\begin{array}{c}\text { Disease-free survival } \\
(\%)\end{array}$} & \multicolumn{4}{|c|}{ Grade $3+$ acute toxicity (\%) } \\
\hline & & 3 years & 5 years & 3 years & 5 years & 3 years & 5 years & GI & GU & Derm. & Acute $\mathrm{NH}$ \\
\hline $\mathrm{JCUH}$ & $\begin{array}{l}50.4 \text { in } 28 \mathrm{fx} \text { and } \\
54 \mathrm{~Gy} \text { in } 30 \mathrm{fx}\end{array}$ & $\begin{array}{l}76 \\
(68-84)^{a}\end{array}$ & $\begin{array}{l}68 \\
(59-79)^{a}\end{array}$ & $\begin{array}{l}85 \\
(78-93)^{a}\end{array}$ & $\begin{array}{l}85 \\
(78-93)^{a}\end{array}$ & $\begin{array}{l}68 \\
(60-78)^{a}\end{array}$ & $\begin{array}{l}59 \\
(49-70)^{a}\end{array}$ & 12 & 0 & 51 & 56.2 \\
\hline ACT $\mathrm{I}^{\mathrm{b}}$ & 45 Gy in $20-25$ fx & 65 & 53 & NA & NA & 39 & 41 & 14 & 3 & 50 & $67^{c}$ \\
\hline $\begin{array}{l}\text { RTOG } \\
98-11\end{array}$ & $\begin{array}{l}45-50.4 \text { Gy in } 1.8 \\
\text { Gy per fx } \pm 5-8 \text { Gy } \\
\text { boost }\end{array}$ & NA & 78 & 90 & 90 & 67 & 60 & 36 & 3 & 49 & 74 \\
\hline ACT $\|^{d, e}$ & 50.4 Gy in $28 \mathrm{fx}$ & NA & 79 & NA & 68 & NA & 69 & 16 & 1 & 48 & 62 \\
\hline \multicolumn{12}{|c|}{$\begin{array}{l}\text { Derm., dematological; fx, fractions; GI, gastrointestinal; GU, genitourir } \\
\text { RTOG, Radiation Therapy Oncology Group. } \\
\text { a'Brackets: } 95 \% \text { confidence interval. } \\
\text { bClassified severity of acute toxicity as 'mild, moderate or severe'. }{ }^{13} \\
\text { c'Severe'. }\end{array}$} \\
\hline
\end{tabular}




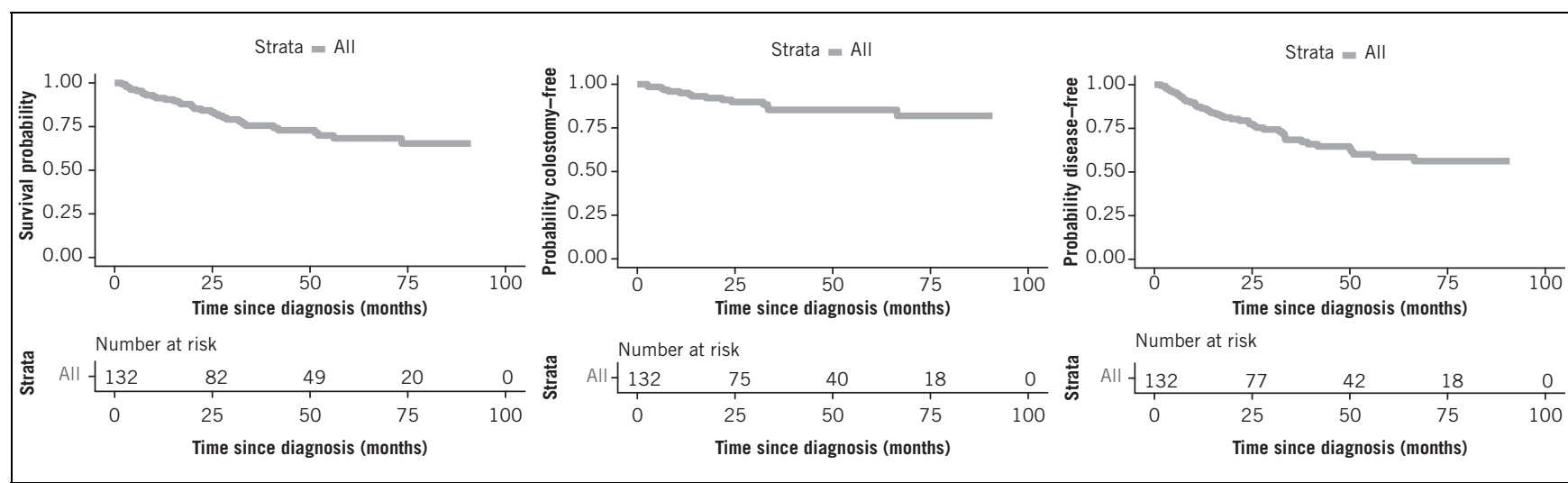

Figure 1 Survival curves representing the Kaplan-Meier estimates of survival: a) overall; b) colostomy-free; c) disease-free.

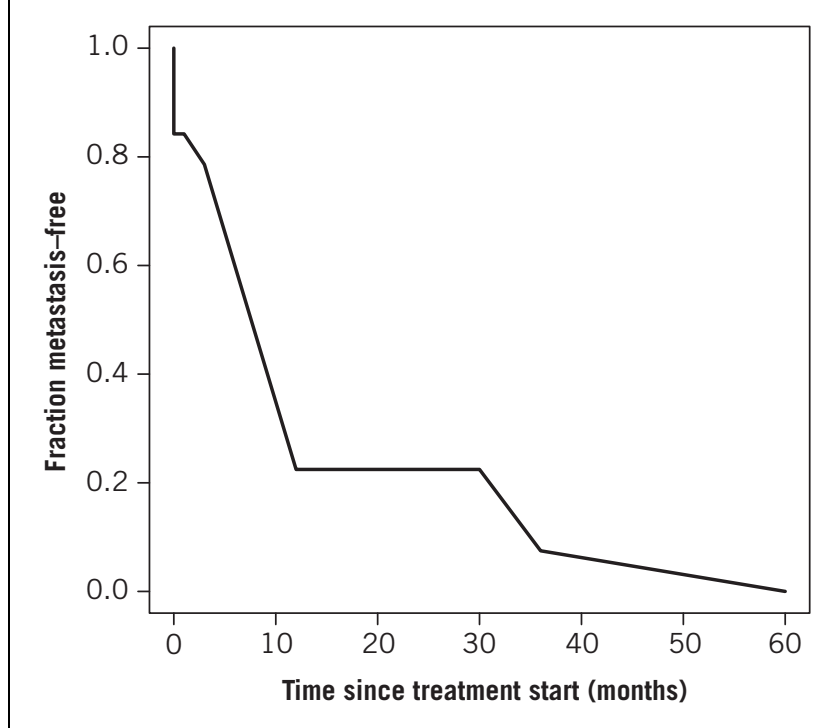

Figure 2 Survival curve representing the Kaplan-Meier estimate of metastasis-free survival. The $y$ axis represents the fraction of those patients who would eventually be found to have a distant metastasis $(n=19)$ who are metastasis-free. The $x$ axis represents the time from initiation of treatment in months. The non-parametric survival curve is calculated using interval-censored data.
$11 \%$ of patients in the toxicity group experienced a grade 3 or 4 chronic toxicity (Table 3 ).

\section{Radiation dose-related outcomes}

Kaplan-Meier estimates of the survival curves for patients who received IMRT radiotherapy with a total dose to the main tumour volume of either 50.4 Gy or 54 Gy are shown in Figure 3. Patients who did not receive the prescribed dose were excluded from this analysis. The five-year survival estimates for patients who received either 50.4 Gy or 54 Gy were $84 \%$ (95\% CI $72-98 \%$ ) and $64 \%$ (95\% CI 46 $88 \%$ ), respectively. The difference between the two groups was not statistically significant $(P=0.061$, hazard ratio, HR, 1.32; 95\% CI 0.98-1.78). Total grade $3+$ acute toxicity (gastrointestinal, genitourinary and dermatological) was 54\% in the 50.4 Gy group and $52 \%$ in the 54 Gy group.

\section{Prognostication}

The assessment of the prognostic factors in our study revealed that in both univariate and multivariate analysis, age at diagnosis $(P=0.02$, HR $1.04 ; 95 \%$ CI 1.01-1.07) and tumour size $(P=0.006$, HR $1.42 ; 95 \%$ CI $1.02-1.98)$ predict shorter survival among patients with anal cancer (Figure 4; Tis not included). However, nodal disease $(P=0.67)$ and tissue differentiation grading $(P=0.41)$ did not significantly influence the survival of these patients in either univariate or multivariate analysis. Male sex was not significantly associated with worse survival on univariate analysis

Table 3 Comparison of patients experiencing grade 3+ chronic toxicity at James Cook University Hospital with patients in the Radiation Therapy Oncology Group 98-11 trial.

\begin{tabular}{|c|c|c|c|c|}
\hline Trial & Overall $^{\mathrm{a}}(\%)$ & Gastrointestinal (\%) & Genitourinary (\%) & Dermatological (\%) \\
\hline $\mathrm{JCUH}$ & 9.4 & 4.7 & 3.1 & 3.1 \\
\hline RTOG 98-11 & 6.9 & 3.2 & NA & 1.6 \\
\hline
\end{tabular}



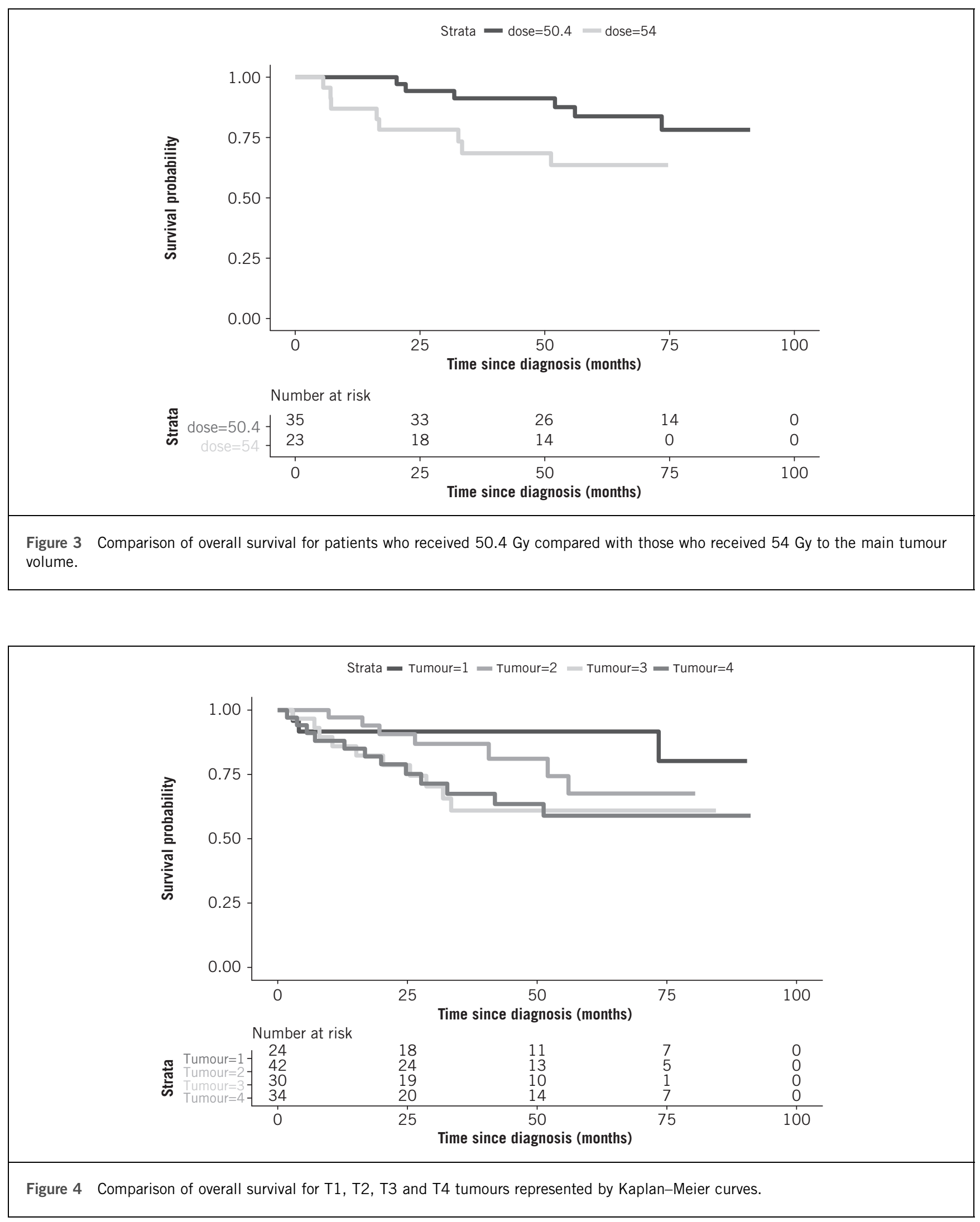
$(P=0.13)$ but was on multivariate analysis $(P=0.04$, HR $2.19 ; 95 \%$ CI $1.02,4.68)$.

\section{Discussion}

A 13-year follow-up of the ACT I trial found a higher rate of non-anal cancer-related deaths in the group receiving chemoradiotherapy compared with those receiving radiotherapy alone. Although chemoradiotherapy showed superior locoregional control over radiotherapy, absolute risk of mortality was not significantly different at 5, 10 and 12 years after beginning treatment. ${ }^{15}$ The Intergroup Radiation Therapy Oncology Group (RTOG) 98-11 trial compared chemoradiotherapy with mitomycin and fluorouracil to chemoradiotherapy with cisplatin and fluorouracil. ${ }^{12}$ In a long-term update of the RTOG 98-11 trial, 200/325 patients $(61.5 \%)$ in the mitomycin arm of the study and 163/324 $(50.3 \%)$ in the cisplatin arm required treatment breaks due to acute toxicity related to chemotherapy. ${ }^{25}$ The ACT II trial also reported high rates of adverse events related to chemoradiotherapy toxicity. Overall, $62 \%$ of patients in the mitomycin group experienced a grade 3 or 4 non-haematological toxicity. ${ }^{11}$

In addition to the immediate adverse effects experienced by the patient, treatment breaks may reduce the efficacy of the treatment, presumably due to accelerated tumour repopulation. ${ }^{26} \mathrm{~A}$ number of studies have also demonstrated a benefit from reduced length of treatment. ${ }^{27-29}$ Taken together, the results of these trials demonstrate that patients receiving chemoradiotherapy are exposed to a large burden of toxicity, which may reduce overall survival and may have the potential to increase the rate of colostomies required for high stool frequency and poor sphincter control.

Dosimetric studies predict a reduction in the radiation exposure to bowel, bladder, genitalia and perianal skin, ${ }^{30-}$ 32 and this benefit has been borne out in several retrospective and prospective studies. ${ }^{16-22}$ In the RTOG 05-29 trial, IMRT led to shorter overall treatment times relative (median 43 days, range 32-59 days), compared with the RTOG 98-11 trial's radiation/fluorouracil/mitomycin arm (median 49 days, range $4-100$ days; $P<0.0001) .{ }^{20,33}$ In our study, 12 patients in the toxicity subset $(18.8 \%)$ required a treatment break of at least one day, while the median treatment time was 37 days, which is less than that in the RTOG 98-11 trial (49 days) and the ACT II trial (38 days). ACT I did not give a median treatment duration. ACT I and ACT II had treatment delays of at least one day in 19 and $15.7 \%$ of their patients, so it is important to point out that we could further reduce our treatment time by reducing the number of delays. Treatment delays may be caused by factors other than acute toxicity, such as patient concordance and illness not related to acute toxicity from radiotherapy.

To our knowledge, the data presented here represent the largest outcome study of patients treated with IMRT for anal cancer. We report low rates of salvage abdominoperineal resection $(8.3 \%)$ and overall, colostomy-free and disease-free survival rates of $68 \%, 85 \%$ and $59 \%$ at five years, respectively, which is comparable or slightly lower than large phase 3 trials that used three-dimensional conformal radiotherapy (Table 2). We found that increasing age at diagnosis was associated with worse survival $(P=0.02)$, although the effect was subtle (HR 1.035). This is in keeping with a large epidemiological study $(n=19,199)$ which found lower five-year survival in patients 65 years or older compared with those between 18 and 65 years in multivariate Cox regression analysis. ${ }^{34}$ Interestingly, this study also found that those living in lower-income areas also had lower five-year survival even when controlling for age and disease stage at presentation (HR 1.09). Given that ours is a single institution catering to a relatively low socioeconomic demographic, it is likely that our survival outcomes will be lower than the national average. As of 2015, average household incomes in the Tees Valley were 15\% below the national average..$^{55}$

We have demonstrated benefit from IMRT compared with chemotherapy and conventional radiotherapy as delivered in the RTOG 98-11 and ACT II trials. We delivered a larger radiation dose to $\mathrm{T} 3$ and $\mathrm{T} 4$ tumours (54, Gy in 30 fractions) while seeing an apparent reduction in acute toxicities. Overall, grade 3 and 4 acute gastrointestinal, genitourinary or dermatological toxicities were experienced by $53 \%$ of our patients. Our patients experienced fewer grade 3 and grade 4 gastrointestinal and genitourinary toxicities than those of RTOG 98-11 and ACT II (Table 2). However, we report a similar rate of grade 3 and 4 dermatological toxicity, possibly because radiation therapy unavoidably passes through skin. Unpublished observations from the oncologist who followed up the toxicity group of this study suggest that the area of affected skin is reduced in IMRT compared with conformal radiotherapy, but the severity of the toxicity is comparable or worse. Some institutions have demonstrated a reduction in dermatological toxicity with IMRT compared with conventional three-dimensional radiotherapy, ${ }^{36,37}$ while others have demonstrated no difference. $^{38}$ In the RTOG 05-29 trial, IMRT resulted in fewer dermatological toxicities relative to RTOG 98-11 (23\% vs $49 \% ; P<0.0001) .{ }^{37}$ Our rates of chronic toxicity were comparable to those of RTOG 98-11 despite IMRT (Table 3).

The larger dose prescribed for $\mathrm{T} 3$ and $\mathrm{T} 4$ tumours did not prevent these patients from continuing to have a worse prognosis relative to those with $\mathrm{T} 1$ and $\mathrm{T} 2$ tumours $(P=0.006$; Figure 4$)$. However, the difference in overall survival between those patients in the toxicity group who received 50.4 Gy and those who received 54 Gy only bordered on significant ( $P=0.061$; Figure 3$)$. Comparison of the total grade $3+$ acute toxicities (gastrointestinal, genitourinary and dermatological) experienced by patients who received 50.4 Gy compared with 54 Gy shows similar rates in both groups (54\% vs $52 \%$, respectively). Furthermore, our rates of acute toxicities are lower than those of previous studies (Table 2), despite the larger radiation dose in our patients. Janssen et al delivered 59 Gy to all tumours regardless of stage and demonstrated exceptional outcomes in terms of acute toxicities (24\% overall) with good tumour control (two-year local control, colostomy-free survival, distant metastases-free survival and overall survival 
rates were $92 \%, 92 \%, 92 \%$, and $88 \%$, respectively). ${ }^{39}$ Given this result, there are grounds for considering an increase in the radiotherapy dose for $\mathrm{T} 3$ and $\mathrm{T} 4$ tumours. Further dose escalation for locally advanced tumours is currently being investigated by the PLATO Anal Cancer Trial $5 .^{40}$

We acknowledge that, as a single-institution study, there are limitations to the applicability of our results. However, given that our institution delivers care to a large and varied demographic spread over both urban and rural areas, these limitations are not likely to negate the importance of our conclusions. The retrospective nature of the survival data means that events which are not recorded in hospital records are absent from our data. As the raw data of the ACT I, ACT II and RTOG 98-11 trials are not available to us, we are not able to make a statistical comparison between the survival and toxicity results from our study and theirs. Furthermore, it is less than ideal that we were not able to prospectively follow-up all patients for toxicity. Instead, we have closely followed up a subset of the total patient group. Conversely, both the overall patient cohort and toxicity subset are large ( $n=132$ and $n=64$, respectively), follow-up was long (median 43 months) and we have obtained a rich dataset due to the meticulous records in electronic hospital databases.

\section{Conclusions}

IMRT is likely to reduce the incidence of acute grade $3+$ gastrointestinal and genitourinary toxicities compared with trials using conventional radiotherapy, while maintaining similar overall, colostomy and disease-free survival. Most patients are treated successfully with salvage abdominoperineal resection for locoregional failure.

\section{References}

1. de Martel C, Ferlay J, Franceschi S et al. Global burden of cancers attributable to infections in 2008: a review and synthetic analysis. Lancet Oncol 2012; 13 (6): 607-615.

2. Islami F, Ferlay J, Lortet-Tieulent J et al. International trends in anal cancer incidence rates. Int J Epidemiol 2017; 46: 924-938.

3. Chaturvedi AK. Beyond cervical cancer: burden of other hpv-related cancers among men and women. J Adolesc Heal 2010; 46(4 Suppl): S20-S26.

4. Lorincz AT, Nathan M, Reuter $\mathrm{C}$ et al. Methylation of HPV and a tumor suppressor gene reveals anal cancer and precursor lesions. Oncotarget 2017; 8 (31): 50,510-50,520.

5. Machalek DA, Poynten M, Jin F et al. Anal human papillomavirus infection and associated neoplastic lesions in men who have sex with men: a systematic review and meta-analysis. Lancet Oncol 2012; 13(5): 487-500.

6. Nigro ND, Vaitkevicius VK, Considine B Jr. Combined therapy for cancer of the anal canal: a preliminary report: 1974. Dis Colon Rectum 1993; 17(3): 354-356.

7. Nigro ND, Vaitkevicius VK, Considine B Jr. Combined therapy for cancer of the anal canal: a preliminary report. Dis Colon Rectum 1974 Jul; 17(3): 354-356.

8. Nigro ND, Seydel HG, Considine B et al. Combined preoperative radiation and chemotherapy for squamous cell carcinoma of the anal canal. Cancer 1983; 51(10): 1,826-1,829.

9. Shridhar R, Shibata D, Chan E, Thomas CR. Anal cancer: current standards in care and recent changes in practice. CA Cancer J Clin 2015; 65(2): 139-162.

10. Houlihan OA, O'Neill BDP. Chemoradiotherapy for anal squamous cell carcinoma. Surgeon 2016; 14(4): 202-212.

11. James RD, Glynne-Jones R, Meadows HM et al. Mitomycin or cisplatin chemoradiation with or without maintenance chemotherapy for treatment of squamous-cell carcinoma of the anus (ACT II): a randomised, phase 3 , open-label, $2 \times 2$ factorial trial. Lancet Oncol 2013; 14(6): 516-524.

12. Ajani JA, Winter KA, Gunderson LL et al. Fluorouracil, mitomycin, and radiotherapy vs fluorouracil, cisplatin, and radiotherapy for carcinoma of the anal canal: a randomized controlled trial. JAMA 2008; 299(16): 1,914-1,921.

13. UKCCCR Anal Cancer Trial Working Party. Epidermoid anal cancer: results from the UKCCCR randomised trial of radiotherapy alone versus radiotherapy, 5-fluorouracil, and mitomycin. Lancet 1996; 348: 1,049-1,054.

14. Flam M, John M, Pajak TF et al. Role of mitomycin in combination with fluorouracil and radiotherapy, and of salvage chemoradiation in the definitive nonsurgical treatment of epidermoid carcinoma of the anal canal: results of a phase III randomized intergroup study. J Clin Oncol 1996; 14(9): 2,527-2,539.

15. Northover J, Glynne-Jones R, Sebag-Montefiore D et al. Chemoradiation for the treatment of epidermoid anal cancer: 13-year follow-up of the first randomised UKCCCR Anal Cancer Trial (ACT I). Br J Cancer 2010; 102: 1,123-1,128.

16. Milano MT, Jani AB, Farrey $\mathrm{KJ}$ et al. Intensity-modulated radiation therapy (IMRT) in the treatment of anal cancer: toxicity and clinical outcome. Int J Radiat Oncol 2005; 63(2): 354-361

17. Salama JK, Mell LK, Schomas DA et al. Concurrent chemotherapy and intensity-modulated radiation therapy for anal canal cancer patients: a multicenter experience. J Clin Oncol 2007; 25(29): 4,581-4,586.

18. Pepek JM, Willett CG, Wu QJ et al. Intensity-modulated radiation therapy for anal malignancies: a preliminary toxicity and disease outcomes analysis. Int $\mathrm{J}$ Radiat Oncol 2010; 78(5): 1,413-1,419.

19. Kachnic LA, Tsai HK, Coen JJ et al. Dose-painted intensity-modulated radiation therapy for anal cancer: a multi-institutional report of acute toxicity and response to therapy. Int J Radiat Oncol 2012; 82(1): 153-158.

20. Kachnic LA, Winter K, Myerson RJ et al. RTOG 0529: A phase II evaluation of dose-painted intensity modulated radiation therapy in combination with 5 -fluorouracil and mitomycin-c for the reduction of acute morbidity in carcinoma of the anal canal. Int J Radiat Oncol Biol Phys 2013; 86(1): 27-33.

21. Bazan JG, Hara W, Hsu A et al. Intensity-modulated radiation therapy versus conventional radiation therapy for squamous cell carcinoma of the anal canal. Cancer 2011; 117(15): 3,342-3,351.

22. Chuong MD, Hoffe SE, Weber J et al. Outcomes of anal cancer treated with definitive IMRT-based chemoradiation. J Radiat Onco/1(2): 165-172.

23. NHS South Tees Hospitals NHS Foundation Trust. The James Cook University Hospital. https://www.southtees.nhs.uk/hospitals/james-cook (cited October 2018).

24. US Department of Health and Human Services, National Cancer Institute. Common Terminology Criteria for Adverse Events (CTCAE) Common Version 4.0. 24 May 2009. https://www.eortc.be/services/doc/ctc/CTCAE_4.03_2010-0614_QuickReference_5x7.pdf (cited October 2018).

25. Gunderson LL, Winter KA, Ajani JA et al. Long-term update of US GI Intergroup RTOG 98-11 Phase III trial for anal carcinoma: survival, relapse, and colostomy failure with concurrent chemoradiation involving fluorouracil/mitomycin versus fluorouracil/cisplatin. J Clin Oncol 2012; 30: 4,344-4,351.

26. Kim JJ, Tannock IF. Repopulation of cancer cells during therapy: an important cause of treatment failure. Nat Rev Cancer 2005; 5(7): 516-525.

27. Graf $R$, Wust $P$, Hildebrandt $B$ et al. Impact of overall treatment time on local control of anal cancer treated with radiochemotherapy. Oncology 2003; 65(1): $14-22$

28. Roohipour R, Patil S, Goodman KA et al. Squamous-cell carcinoma of the anal canal: predictors of treatment outcome. Dis Colon Rectum 2008; 51(2): 147-153.

29. Ben-Josef E, Moughan J, Ajani J et al. The impact of overall treatment time on survival and local control in anal cancer patients: a pooled data analysis of RTOG trials 8704 and 9811. J Clin Oncol 2010; 28(34): 5,061-5,066.

30. Chen Y-J, Liu A, Tsai PT et al. Organ sparing by conformal avoidance intensitymodulated radiation therapy for anal cancer: dosimetric evaluation of coverage of pelvis and inguinal/femoral nodes. Int J Radiat Oncol Biol Phys 2005; 63(1): 274-281.

31. Lin A, Ben-Josef E. Intensity-modulated radiation therapy for the treatment of anal cancer. Clin Colorectal Cancer 2007; 6(10): 716-719.

32. Menkarios C, Azria D, Laliberté B et al. Optimal organ-sparing intensity-modulated radiation therapy (IMRT) regimen for the treatment of locally advanced anal canal carcinoma: a comparison of conventional and IMRT plans. Radiat Oncol 2007; 2(1): 41.

33. Herman JM, Thomas CR. RTOG 0529: Intensity modulated radiation therapy and anal cancer, a step in the right direction? Int J Radiat Oncol Biol Phys 2013; 86(1): 8-10. 
34. Bilimoria KY, Bentrem DJ, Rock CE et al. Outcomes and prognostic factors for squamous-cell carcinoma of the anal canal: analysis of patients from the national cancer data base. Dis Colon Rectum 2009; 52(4): 624-631.

35. Tees Valley Unlimited. Tees Valley Economic Assessment 2015/16. Stockton-onTees: Tees Valley Unlimited; 2016.

36. Chuong MD, Freilich JM, Hoffe SE et al. Intensity-modulated radiation therapy vs. $3 \mathrm{~d}$ conformal radiation therapy for squamous cell carcinoma of the anal canal. Gastrointest Cancer Res 2013; 6(2): 39-45

37. Kachnic LA, Winter K, Myerson RJ et al. RTOG 0529: a phase 2 evaluation of dose-painted intensity modulated radiation therapy in combination with 5 fluorouracil and mitomycin-C for the reduction of acute morbidity in carcinoma of the anal canal. Int J Radiat Oncol Biol Phys 2013; 86(1): 27-33.
38. Saarilahti K, Arponen P, Vaalavirta L, Tenhunen M. The effect of intensitymodulated radiotherapy and high dose rate brachytherapy on acute and late radiotherapy-related adverse events following chemoradiotherapy of anal cancer. Radiother Oncol 2008; 87(3): 383-390.

39. Janssen $\mathrm{S}$, Glanzmann $\mathrm{C}$, Bauerfeind $\mathrm{P}$ et al. Clinical experience of

SIB-IMRT in anal cancer and selective literature review. Radiat Oncol 2014; 9 (1): 199.

40. Sebag-Montefiore D, Adams R, Bell S et al. The development of an umbrella trial (PLATO) to address radiation therapy dose questions in the locoregional management of squamous cell carcinoma of the anus. Int J Radiat Oncol 2016 96(2): E164-E165 